\title{
Combination of Radiofrequency Ablation and Glycated Chitosan as Treatment on a Syngeneic Breast Tumor Model
}

\author{
HSIN-YU CHIU ${ }^{1 *}$, JYH-DER LEU ${ }^{2 *}$, CHUN-YUAN CHANG ${ }^{1}$, YI-JANG LEE ${ }^{1,3}$ and WEI R. CHEN ${ }^{4}$ \\ ${ }^{1}$ Department of Biomedical Imaging and Radiological Sciences, \\ National Yang-Ming University, Taipei, Taiwan, R.O.C.; \\ ${ }^{2}$ Division of Radiation Oncology, Taipei City Hospital RenAi Branch, Taipei, Taiwan, R.O.C.; \\ ${ }^{3}$ Biophotonics and Molecular Imaging Research Center (BMIRC), \\ National Yang-Ming University, Taipei, Taiwan, R.O.C.; \\ ${ }^{4}$ Biophotonics Research Laboratory, Center for Interdisciplinary Biomedical Education and Research, \\ University of Central Oklahoma, Edmond, OK, U.S.A.
}

\begin{abstract}
Aim: Effects of radiofrequency ablation (RFA) combining immunoadjuvant glycated chitosan $(G C)$ on tumor control and potent cytokine responses were investigated in a syngeneic breast tumor model. Materials and Methods: Murine $4 T 1$ breast carcinoma cells harboring the luciferase reporter gene were used to evaluate the tumor growth rate and metastasis in vivo using bioluminescent imaging. Plasma of RFA/GC-treated tumor-bearing mice was collected for ex vivo cytotoxicity analysis and mouse chemokine array assays. Results: Tumor growth and systemic metastasis were suppressed by combined RFA and GC when tumor size reached $300 \mathrm{~mm}^{3}$, not detected, however, when tumor size reached $800 \mathrm{~mm}^{3}$. The survival rate of mice bearing small tumors was also higher than that of large ones after RFA-GC treatment. Plasma extracted from RFA-GC-treated small tumor-bearing mice exhibited cytotoxicity on cultured $4 T 1$ cells. Moreover, reduced tumor growth-related cytokines and increased antitumor-related cytokines were detected in the plasma collected. Conclusion: RFA combining GC could control tumor progression with induced potent antitumor cytokine responses.
\end{abstract}

Combination of different therapeutic approaches is commonly applied in cancer treatment. Hyperthermia is one of the methods

\footnotetext{
*These Authors contributed equally to this study.
}

Correspondence to: Yi-Jang Lee, Ph.D., Department of Biomedical Imaging and Radiological Sciences, National Yang-Ming University, No. 155, Sec. 2, Linong St. Beitou District, 112, Taipei, Taiwan, R.O.C. Tel: +886 228267189, Fax: +886 228201095, e-mail: yjlee2@ym.edu.tw

Key Words: Glycated chitosan, radiofrequency ablation, syngeneic tumor model, breast cancer, antitumor, cytokine responses. using local heat for tumor control via various physical approaches and modalities, such as radiofrequency (RF) and focused ultrasound (FUS). Hyperthermia is usually combined with radiotherapy and chemotherapy because it may change the tumor's microenvironment and increase blood flow for oxygenation to provide radio-chemosensitization $(1,2)$. Although the therapeutic efficacy is diverse, combined hyperthermia and other treatments have been applied in the treatment of melanoma, cervical cancer, head and neck cancer, breast cancer, brain cancer, lung cancer, liver cancer and bladder cancer (3-6). Although hyperthermia can also induce cell killing effects at high temperature range, its tumor suppressive effects remain to be addressed using mild hyperthermia.

Radiofrequency ablation (RFA) depends on an electrical generator that hooks a needle electrode to deliver high frequency alternative currents by penetrating through skin to the tumor mass. RFA is regarded the most commonly used modality for tumorous thermal ablation and applied in the treatments of liver cancer, lung cancer, renal carcinomas, breast cancer and prostate cancer $(7,8)$. RFA-mediated elevation of effective temperature for tumor control may depend on tumor types. For instance, it aims to reach $50^{\circ} \mathrm{C}$ to $100^{\circ} \mathrm{C}$ to kill liver tumors (9). Alternatively, there was a report that employed $46.8^{\circ} \mathrm{C}$ to $79^{\circ} \mathrm{C}$ for breast cancer treatment (10). The temperature can also be adjusted between $42^{\circ} \mathrm{C}$ and $44^{\circ} \mathrm{C}$ to avoid damage of normal tissues surrounding targeted tumors (11). In addition to heat effect, RFA has been reported to activate tumor specific T-cell responses for cancer therapy (12). RFA also induces antitumor immune responses by modulating the immune system of metastatic liver patients (13).

Adjuvants are immunological agents that modify or augment an immune response, usually to a vaccine, without having any specific antigenic effect on their own (14). The most common adjuvants for human application today are aluminum hydroxide and monophosphoryl lipid A (MPL) 
(14). However, their application has not been widely used in cancer therapy. Glycated chitosan (GC) is derived from chitosan, a linear polysaccharide composed of Dglucosamine and $\mathrm{N}$-acetyl-D-glucosamine through $\beta$-[1-4] linkage (15). This modification improves the water solubility of chitosan for various biomedical applications (16). Several lines of evidence have shown that GC is an immunoadjuvant agent that can target primary and metastatic tumors formed in animal and human patients (16-18). By combining laser photothermal and photodynamic therapy, GC is believed to induce immunological responses to attack tumors, so called laser immunotherapy (LIT) $(16,19-23)$. Additionally, GC has been combined with nanomaterials, such as single-walled carbon nanotubes (SWNTs), and used for improving the immuno-modified effects of GC on tumor suppression through photothermal therapy. Although the optical-mediated induction of immune responses has been broadly used in these researches, photon-based therapy remains uncommonly used in clinic. RFA has been widely used as a modality of hyperthermia in hospitals. It is of interest to investigate whether GC can combine with RFA for better tumor control.

In this study, we combined GC and RFA to treat a murine breast cancer using a syngeneic tumor model. This combination revealed that small tumors were more sensitive than larger tumors. Plasma extracted from whole blood of mice bearing small tumors, but not large tumors, with RFAGC treatment also showed higher toxicity on cultured 4T1 cells than that from individual treatment. Moreover, chemokine array analysis demonstrated that several cytokines related to tumor development were reduced in plasma. Therefore, the current results support that GC combined with RFA would be a novel strategy for breast cancer control.

\section{Materials and Methods}

Cell culture and chemicals. 4T1 murine breast carcinoma cells with stably transfected multi-cistronic reporter genes have been reported previously, so called 4T1_PB3R (24). Cells were cultured in RPMI1640 medium (Gibco; Invitrogen Inc., Carlsbad, CA, USA) containing $10 \%$ fetal bovine serum (FBS; HyClone; Thermo, Waltham, MA, USA), $1 \%$ penicillin-streptomycin solution (Caisson Laboratories Inc., North Logan, UT, USA) and 1\% l-glutamine (200 mM) (SigmaAldrich Co., St. Louis, MO, USA). Cells were cultured in a $37^{\circ} \mathrm{C}$, humidified incubator containing $95 \%$ air and $5 \% \mathrm{CO}_{2}$ and passaged every 2 days. Preparation of glycated chitosan $(10 \mathrm{mg} / \mathrm{ml}$, dissolved in deionized distilled water) has been described previously (16). It was then stored in the refrigerator until used.

Syngeneic breast tumor model. The immune competent female $\mathrm{Balb} / \mathrm{C}$ mice were used for establishing the syngeneic tumor model (National Laboratory Animal Center, Taipei, Taiwan). 4T1_PB3R tumor cells $\left(1 \times 10^{6}\right.$ in $100 \mu$ l phosphate-buffered saline $)$ were subcutaneously injected into the upper backs of 4-week-old mice. Tumors were formed after one week post-injection and their dimensions were measured using caliper twice a week. The tumor volume was determined by the equation: volume=length $(\mathrm{mm}) \times$ width $(\mathrm{mm})^{2} / 2$. The animal experiments had been reviewed and approved by the Institutional Animal Care and Use Committee (IACUC) of National Yang-Ming University (approval number: 1040722).

Radiofrequency ablation with GC. The tumor-bearing mice were anesthetized and placed on an aluminum plate attached with an electricity-conducting pad. An RFA electrode (a cool-tip RF Ablation Electrodes with cool-tip ${ }^{\mathrm{TM}}$, ACT1510; COVIDIEN, Minneapolis, $\mathrm{MN}$, USA) was penetrated into the middle of the tumors. RFA treatments were done using a radiofrequency generator (CTRF117; COVIDIEN). The temperature was set between $42^{\circ} \mathrm{C}$ and $44^{\circ} \mathrm{C}$ for 10 seconds of treatment. For combined treatment, $100 \mu \mathrm{l}$ of GC solution $(10 \mathrm{mg} / \mathrm{ml})$ was intratumorally injected into the center of tumor at three random peripheral positions of each tumor using a $27-G$ needle. The same strategy was also used for GC treatment alone. The tumor responses were then examined daily.

In vivo bioluminescent imaging. Tumor-bearing mice were intraperitoneally injected with $150 \mathrm{mg} / \mathrm{kg}$ D-luciferin (Caliper Co., Hopkinton, MA, USA) for $15 \mathrm{~min}$ and anesthetized with $2 \%$ isofluorane in a small cabinet. Mice were then removed from the cabinet and placed into the chamber of IVISTM50 system (Xenogen Co., Alameda, CA, USA) for detecting the luminescent signals. Regions of interest were drawn around whole body and luminescent signal was quantified by the number of photons detected per second (photons $/ \mathrm{sec} / \mathrm{cm}^{2} / \mathrm{sr}$ ). Steradian (sr) represents the International System of Units (SI) of solid angle (25).

Isolation of plasma. Tumor-bearing mice were sacrificed using cervical dislocation after different treatments. Blood was immediately collected from cardiac puncture using a $26 \mathrm{G}$ needle. Extracted blood was immediately centrifuged using $1,300 \mathrm{rpm}$ at $4^{\circ} \mathrm{C}$ for 10 minutes. The supernatant was then collected and centrifuged again. The plasma was then passed through a $0.22 \mu \mathrm{m}$ Super Membrane Low Protein Binding filter (PALL Inc., Port Washington, NY, USA).

Cell viability assay. Plasma was diluted ten-fold by culture medium and added into a 96-well plate seeded with 4T1_PB3R cells and incubated under $37^{\circ} \mathrm{C}$ for 2 days. After removal of the supernatant, $1 \mathrm{mg} / \mathrm{ml}$ 3-[4,5-dimethylthiazol-2-yl]-2,5-diphenylterazoliumbromide (MTT) solution (Sigma-Aldrich) was mixed with serum-free medium and added to each well, incubated at $37^{\circ} \mathrm{C}$ for another $4 \mathrm{~h}$. Dimethyl sulfoxide (DMSO) was then added to dissolve crystals. The plate was then quantified using an ELISA reader (Bio-Tek Instruments, Winooski, VT, USA) with a light absorption at $570 \mathrm{~nm}$.

Assay for chemokines in plasma. All monoclonal capture antibodies and cytokines were obtained from RayBiotech Inc. (Norcross, GA, USA) and assay procedures were followed according to the manufacturer's instructions. Plasma was obtained as described above. Ten-fold diluted plasma was incubated with the Mouse Chemokine Array C1 (RayBiotech Inc.) in blocking buffer at $4^{\circ} \mathrm{C}$ overnight. The secondary antibody, conjugated with horseradish peroxidase, was then replaced with the diluted plasma and the membrane was agitated for $2 \mathrm{~h}$ at room temperature. The membrane was rinsed with Western lightning plusenhanced chemiluminescence (ECL) (Perkin-Elmer Inc., Waltham, MA, USA) and chemoluminescent signals were detected using the LAS-4000 gel imaging system (GE Healthcare Inc., Wauwatosa, WI, 


\section{A}

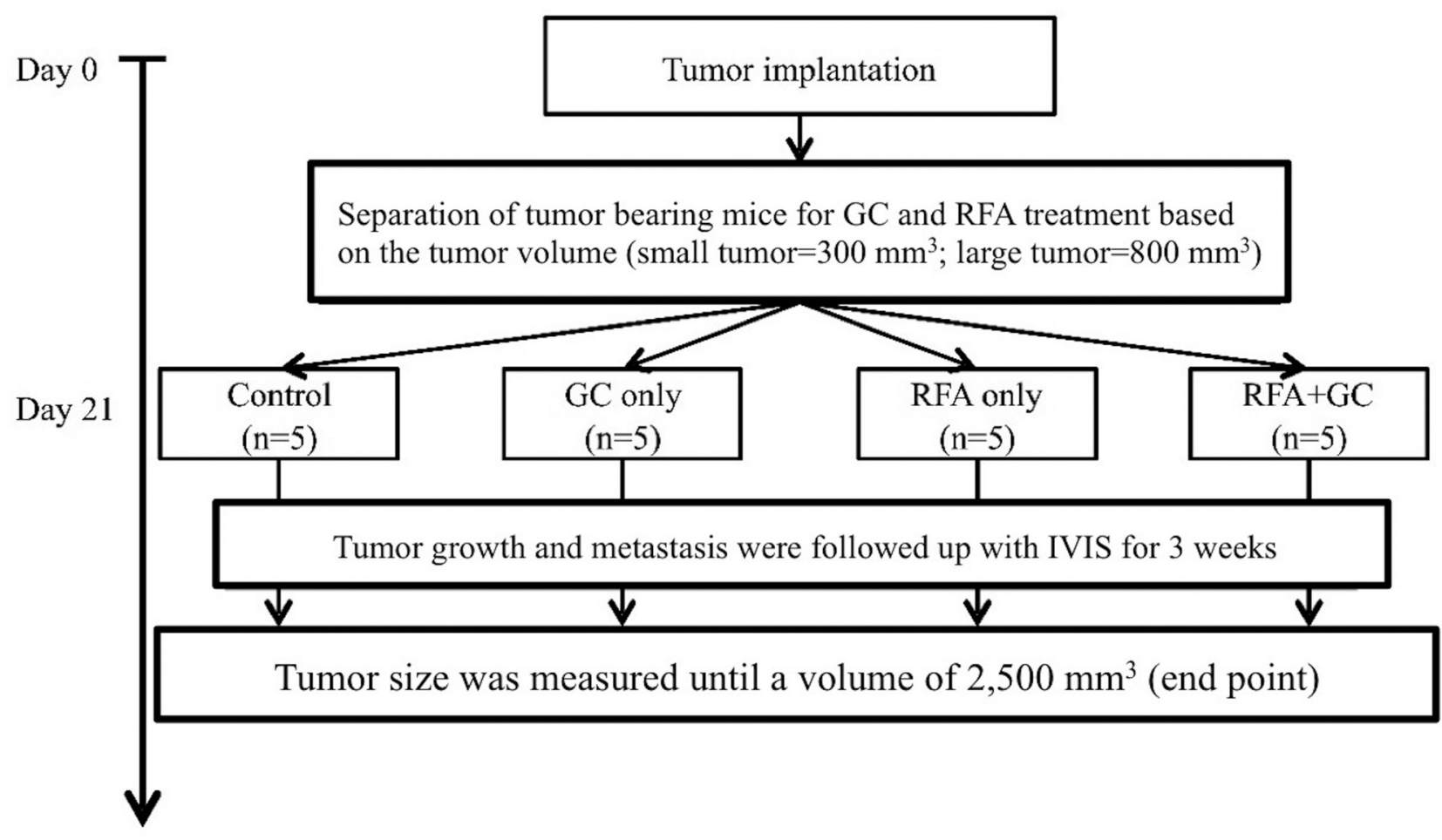

B

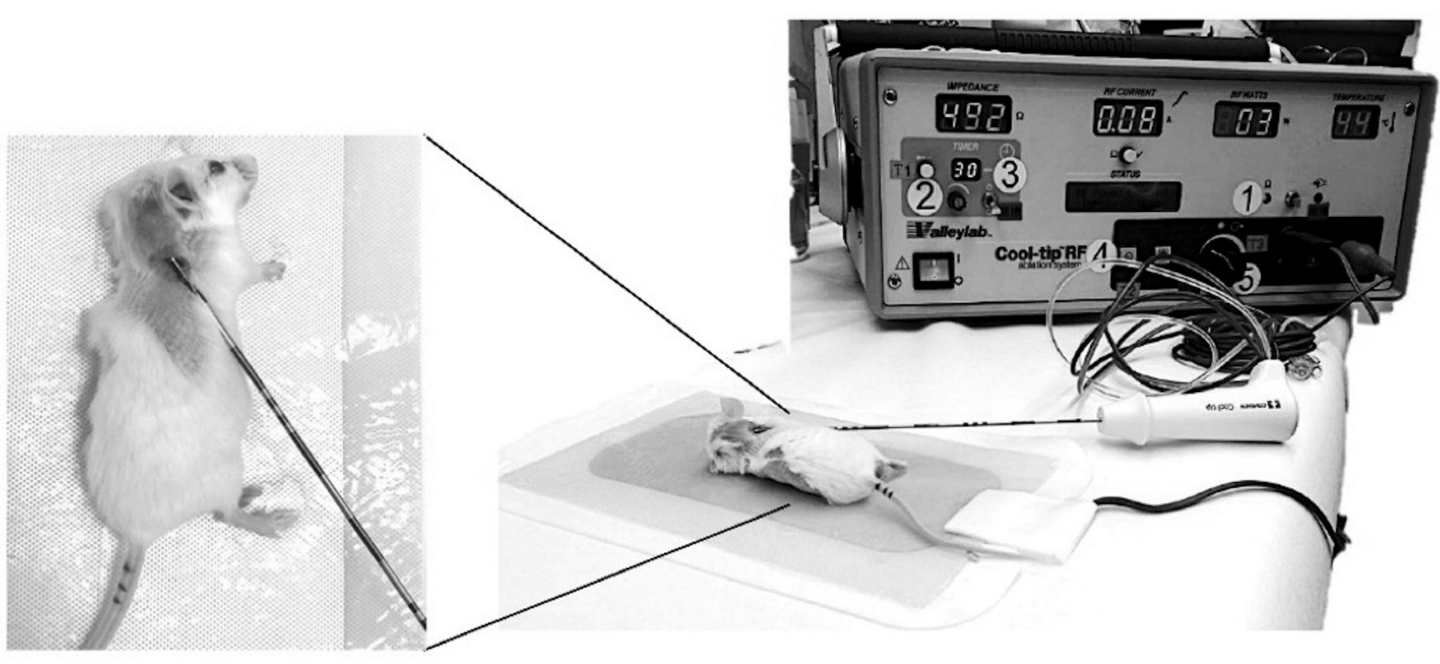

Figure 1. Outlines of experimental designs for GC and RFA treatments on the syngeneic breast tumor model. (A) Study's flowchart; (B) RFA equipment and the electrode insertion into the tumor mass of tumor-bearing mice. GC, Glycated chitosan; RFA, radiofrequency ablation.

USA). The dot densities were quantified using ImageJ software (version 1.46; https://imagej.nih.gov/ij/download.html).

Statistical analysis. Experimental data are presented as the mean of three independent experiments \pm standard deviation (S.D). Data were analyzed with Student's $t$-test or two-way ANOVA (for animal experiments) and $p<0.05$ indicated statistical significance.

\section{Results}

The experimental flowchart for treatment of syngeneic breast tumor model by combining GC and RFA. Tumor-bearing mice were grouped into control, GC-treated only, RFAtreated only and combination. Additionally, treatments were 

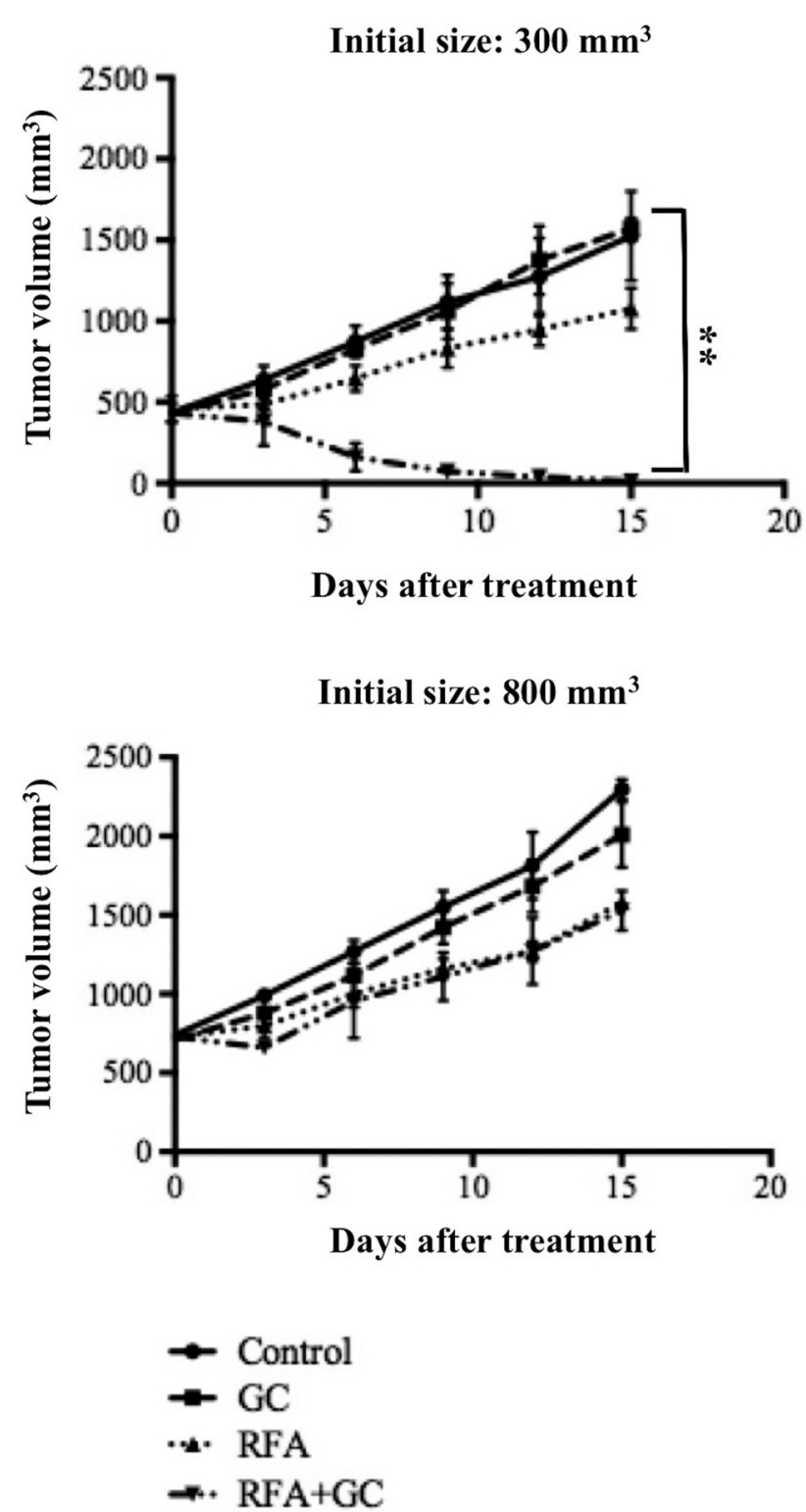

Figure 2. Comparison of tumor growth rates among control, GC, RFA and RFA-GC treatments. Upper and lower panels: Initial tumor volume was $300 \mathrm{~mm}^{3}$ and $800 \mathrm{~mm}^{3}$ for treatment, respectively. $* * p<0.005 . \mathrm{GC}$, glycated chitosan; RFA, radiofrequency ablation.

initiated when tumor volume reached $300 \mathrm{~mm}^{3}$ or $800 \mathrm{~mm}^{3}$. The tumor progression was then monitored using bioluminescent imaging and caliper (Figure 1A). The RF instrument and the position of electrode on implanted tumor are shown in Figure 1B.

Effects of GC and RFA on the tumor growth rate. Tumor growth was determined by measuring the tumor volume at different time points following treatment. When tumor volume initially reached $300 \mathrm{~mm}^{3}$, GC combined with RFA treatment exhibited dramatic tumor killing effects compared to GC or RFA individual treatment or untreated control (Figure 2A). On the other hand, the same treatments on $800 \mathrm{~mm}^{3}$ tumors showed little tumor suppressive effects (Figure 2B). These results suggested that a combination of GC and RFA could diminish the growth of small tumors but not that of large tumors.

Effects of GC and RFA on tumor metastasis. Because the combined GC and RFA treatment could suppress tumor growth at primary site, we were interested in further investigating whether tumor metastasis could be repressed accordingly. To this end, we used bioluminescent imaging to track the development of 4T1 tumors harboring luciferase reporter genes in vivo (see Materials and Methods). The results showed that, in animals with small tumors, tumor growth was completely suppressed and remote metastasis was not detected after RFA-GC combined treatment compared to control and separate treatments (Figure 3A). However, the growth and metastases of animals with large tumors were not suppressed by any of these treatments (Figure 3B). The photon fluxes were also quantified and compared among these regimes (Figure 3C and 3D). These data suggest that combined GC and RFA treatment could significantly suppress tumor growth and subsequent remote metastases of small syngeneic tumors but not large ones.

Effects of GC and RFA on survival of tumor-bearing mice. We next examined the survival of these mice using different treatments. Our results showed that mice bearing small tumors treated with RFA-GC could survive longer than untreated control or mice treated by RFA or GC alone (Figure 4). For large tumorbearing mice, survival rates showed no significant differences among the animals under different treatments (Figure 4). Actually, the RFA-GC-treated small tumor-bearing mice remained tumor-free for over 4 months. Therefore, it appeared that small tumors were more sensitive to combined GC and RFA treatment, with the animals surviving much longer.

Blood plasma extracted from combined GC and RFA-treated tumor-bearing mice exhibited cytotoxicity effects. To further investigate whether the tumoricidal effects caused by combination of GC and RFA treatment is associated with immunological responses, blood plasma was extracted from tumor-bearing mice (both small and large tumors) at 3 weeks after various treatments. The plasma was diluted in culture medium and added to cultured 4T1 cells in a 96-well plate for the MTT assay (Figure 5A). Results showed that plasma of mice bearing small tumors exhibited stronger cytotoxicity than that of mice bearing large tumors after RFA-GC treatment (Figure 5B). Therefore, the GC and RFA combined treatment might induce stronger immune responses in mice with small tumors than those with large tumors. 


\section{A Small tumor B Large tumor}

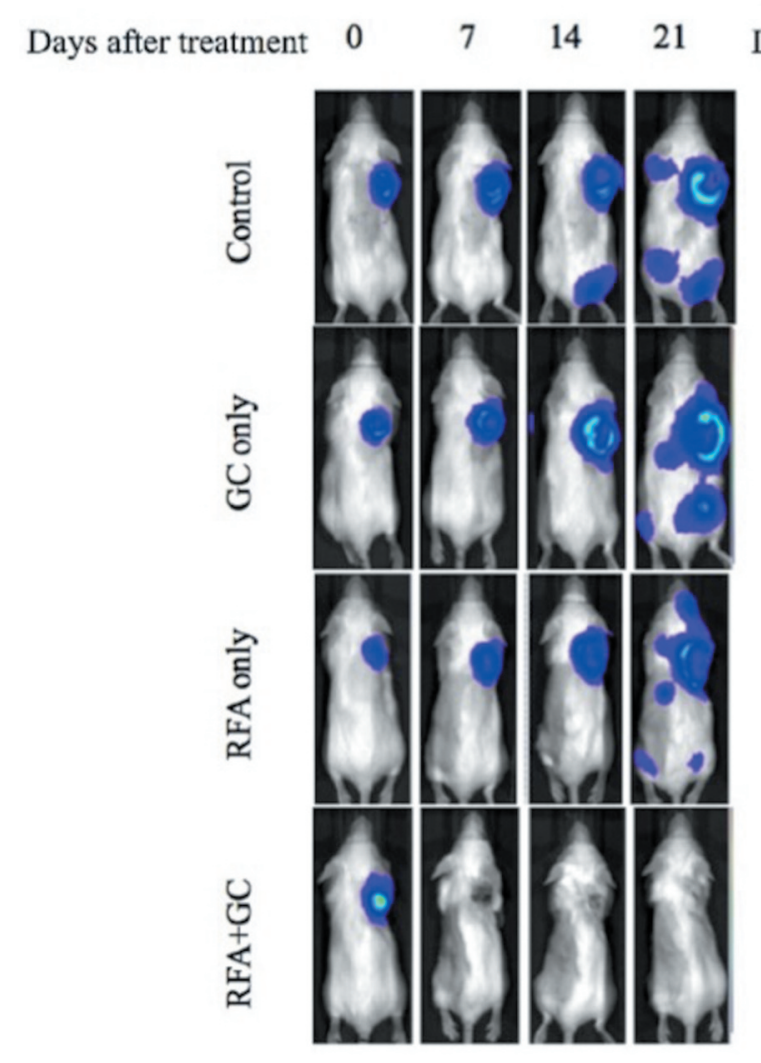

C

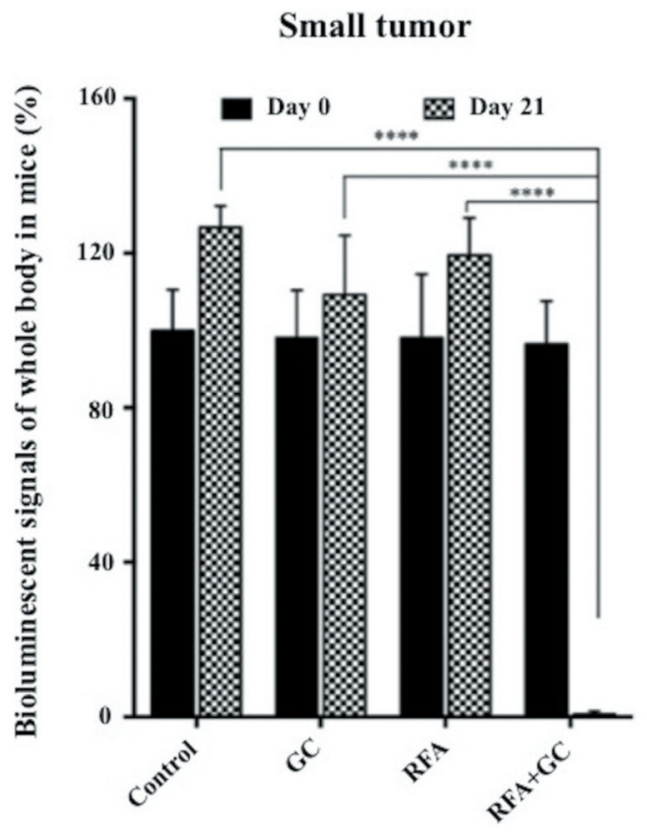

$\begin{array}{lllll}\text { Days after treatment } & 0 & 7 & 14 & 21\end{array}$

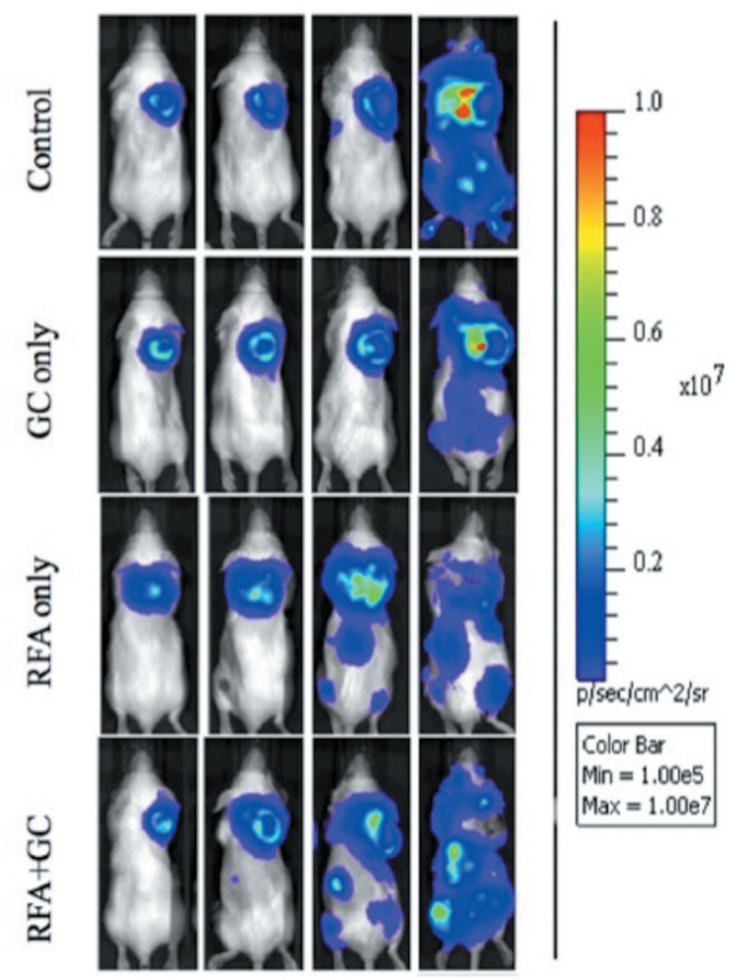

D
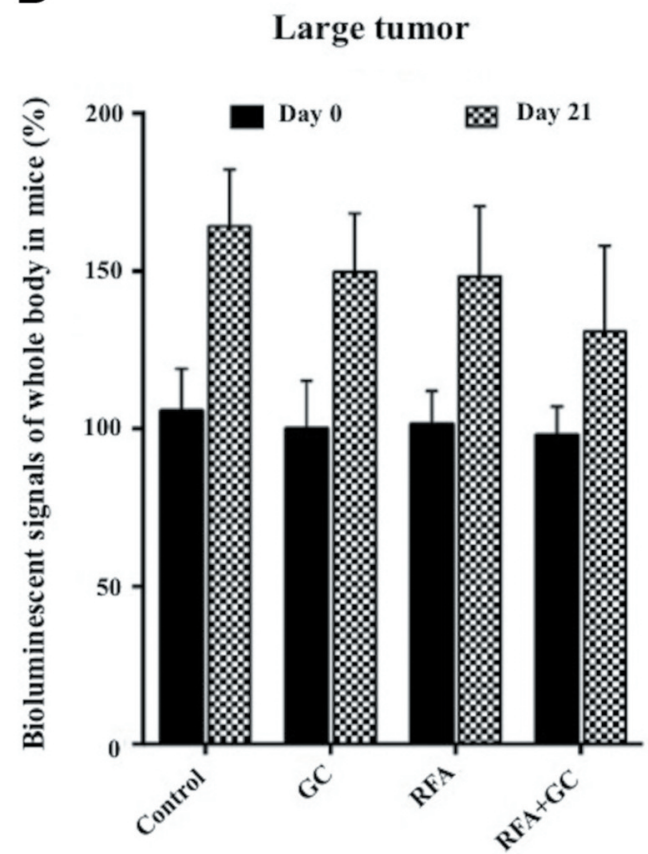

Figure 3. Bioluminescent imaging of tumor progression in tumor-bearing mice.4T1_PB3R cells with luciferase reporter genes were implanted and monitored in vivo. (A) Initial small tumors; $(B)$ Large tumors; $(C)$ and $(D)$ Represent the quantification of photon flux in different treatments of $(A)$ and $(B)$, respectively. $* * * *<0.0001$. GC, Glycated chitosan; RFA, radiofrequency ablation. 
Expression of immunologically-related molecules in plasma extracted from RFA-GC-treated tumor-bearing mice. To understand if any immune responses could be modulated by combined GC and RFA, we used the Mouse Chemokine Array $\mathrm{C} 1$ to analyze the expression of immune-related cytokines in plasma after treatment. Twenty-five different plasma cytokines were detected after tumor-bearing mice when treated with GC, RFA or RFA-GC. The patterns of chemokine array staining were acquired and visualized using a gel imaging system (Figure 6A). After densitometric quantification, fifteen of these chemokines exhibited signals sufficient to be quantified. Compared to control, CXCL13 (B lymphocyte chemoattractant (BLC)) and CCL2 (monocyte chemoattractant protein-1 (MCP-1)) were induced by RFA but were suppressed by RFA-GC combined treatment (Figure 6B). On the other hand, CCL20 (macrophage inflammatory protein 3 alpha (MIP-3- $\alpha)$ ) and CXCL4 (platelet factor 4 (PF-4)) were boosted by combined RFA-GC treatment to different levels, no matter whether they were affected by RFA alone or not (Figure 6B). Although several cytokines, such as CCL27 (cutaneous T-cell-attracting chemokine (CTACK)), CCL11 (Eotaxin-1), CXCL1 (KC), monocyte chemotactic protein-5 (MCP-5), MIP-2 and CCL-5 (regulated on activation, normal T cell expressed and secreted (RANTES)), were also induced by RFA-GC combined treatment they showed little difference from that in RFA treatment alone. Taken together, the current analysis suggested that RFA-GC treatment might change the expression of cytokines in blood for modulating potent immune response to suppress tumor growth.

\section{Discussion}

GC, when combined with photothermal therapy, has shown great promise in the treatment of aggressive DMBA-4 breast tumors in rats with significant antitumor immune response in cured rats that completely resisted repeated tumor challenges. Furthermore, the transfer of splenocytes from cured DMBA-4 breast tumor-bearing rats provided $100 \%$ immunity in the naïve recipients against the same tumor cells, demonstrating that LIT induced adaptive immunity. In this study, we used RFA, an FDA approved minimal invasive therapy for soft tissue tumors, followed by GC to investigate the effects of tumor suppression in a syngeneic breast tumor model (8). Surprisingly, this combination not only eradicated tumor growth at the primary site but also the remote metastasis in mice with small tumors as compared to untreated controls or mice under GC or RFA treatment alone. However, the combined treatment did not show better tumor suppressive effect than RFA alone in mice with large tumors at the time of treatment. Several reports using RFA for human breast cancer treatment usually select an average tumor volume from $0.775 \mathrm{~cm}$ to $2.08 \mathrm{~cm}$ or at early stage (8, 26-31). It is reasonable that heat transduction should be more efficient in small tumors. Additionally, it has been reported that pre-heating at $37^{\circ} \mathrm{C}$ or $43^{\circ} \mathrm{C}$ can enhance cell sensitivity to bleomycin (BLM) or cis-diammine-dichloroplatinum(II) (cis-DDP) (32). Although GC is not regarded a chemotherapeutic agent, pre-heating a small syngeneic tumor followed by GC injection also exhibited apparent tumor suppression. To the best of our knowledge, this is the first report showing that pre-heating treatment using RFA combined with a non-chemotherapeutic agent can suppress tumor growth. Based on the immunoadjuvant property of $\mathrm{GC}$, enhancement of immune responses by this combination could be one of the effects to ablate tumor growth.

Moderate hyperthermia can prevent normal tissues from complications; however, the trade-off effect could be inefficient therapy. In fact, hyperthermia is an adjuvant approach for conventional radiotherapy and chemotherapy (33-36). Accumulated evidence supports that hyperthermia combining radio-chemotherapy can lead to better local control and patient survival with different types of high-risk cancers $(4,37)$. Although GC is not considered as a chemotherapeutic agent, it may alter the immune responses in tumor microenvironment after hyperthermia. Hyperthermia has been believed to induce immunity due to induction of heat shock proteins (HSPs) and local fever (38-40). A recent report has also shown that hyperthermia regulates the interaction between HSPs and mismatch repair (MMR) proteins to influence the cisplatin sensitivity of MMR-deficient/proficient colon cancer cell lines (41). On the other hand, abundant expression of HSPs in tumors correlates to the viability of cancer cells and resists to hyperthermia-induced cytotoxicity $(42,43)$. Because GC combined with RFA exhibited dramatic tumor suppressive effects compared to RFA alone in small tumors, it is of interest to further investigate whether GC can affect the expression of HSPs in the future.

One of the interesting results was that the plasma obtained from RFA-GC-treated mice with small tumors exhibited significant cytotoxicity in cultured 4T1_PB3R cells. Although little is known about the underlying mechanism, the current findings are relevant to those of a previous report that hyperthermia may augment specific immune reaction via antibody-complement cytotoxicity on tumor cells (44). Here, by using chemokine array analysis, it was found that cytokines, including CXCL13 (BLC) and CCL2 (MCP-1), could be affected by RFA. However, the expression of these cytokines was reversely expressed by RFA-GC treatment. CXCL13 and CCL2 were overexpressed in breast cancer and associated with lung metastasis of $4 \mathrm{~T} 1$ cells, respectively $(45,46)$. This finding suggests that GC combining RFA could repress tumor-related cytokines. On the other hand, the most apparent RFA-GC-induced cytokines were CCL20 and CXCL4, that could boost CD8 ${ }^{+} \mathrm{T}$-cell-dependent antitumor response and inhibit angiogenesis to control tumor invasion, respectively $(47,48)$. Moreover, several detected cytokines 

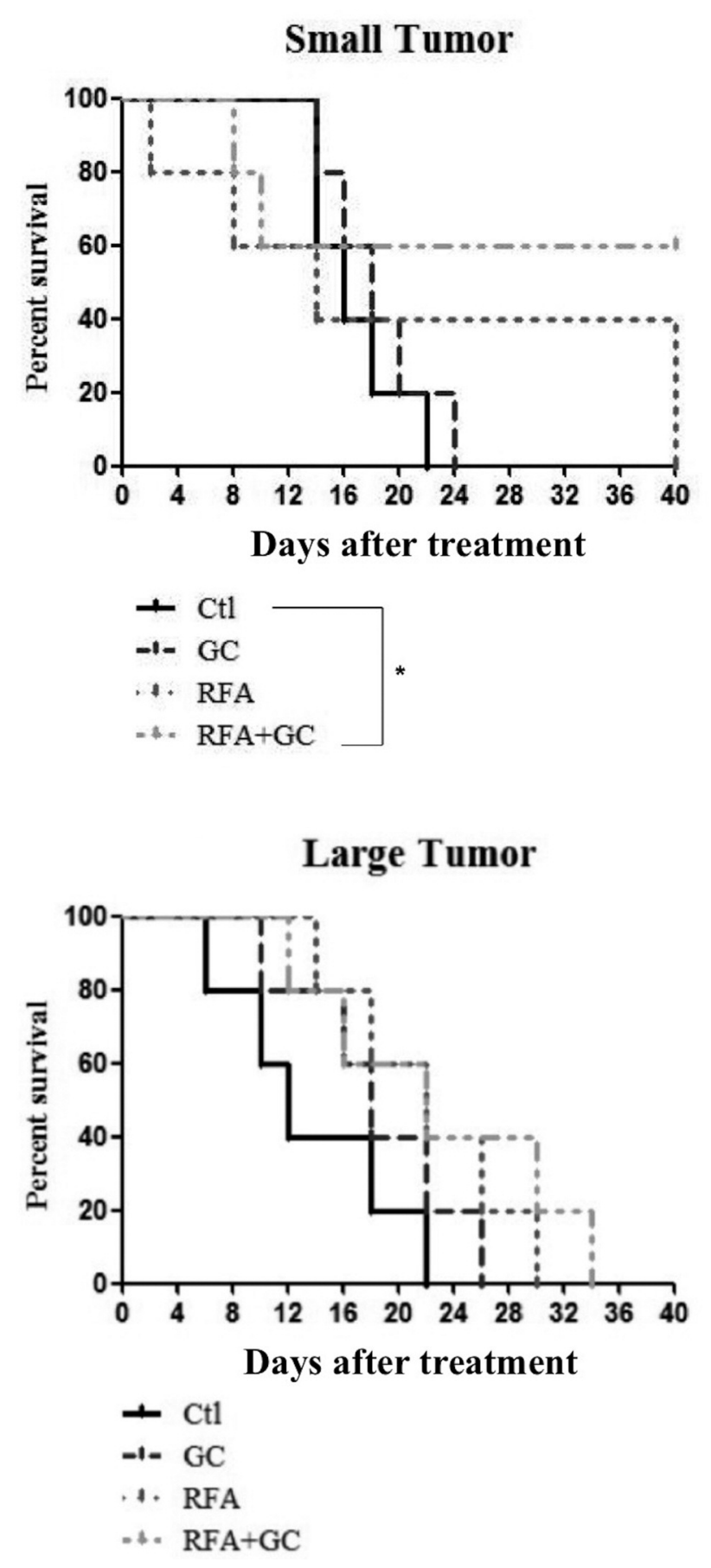

Figure 4. Kaplan-Meier plot for determining the survival of tumorbearing mice after GC and RFA treatments. Statistical analysis was performed using the log-rank test. ${ }^{*} p<0.05$. GC, Glycated chitosan; $R F A$, radiofrequency ablation; $C t l$, control.

were induced by RFA or RFA-GC combined treatment to similar levels. Whether they also play important roles to influence the therapeutic efficacy of RFA-GC combination
A

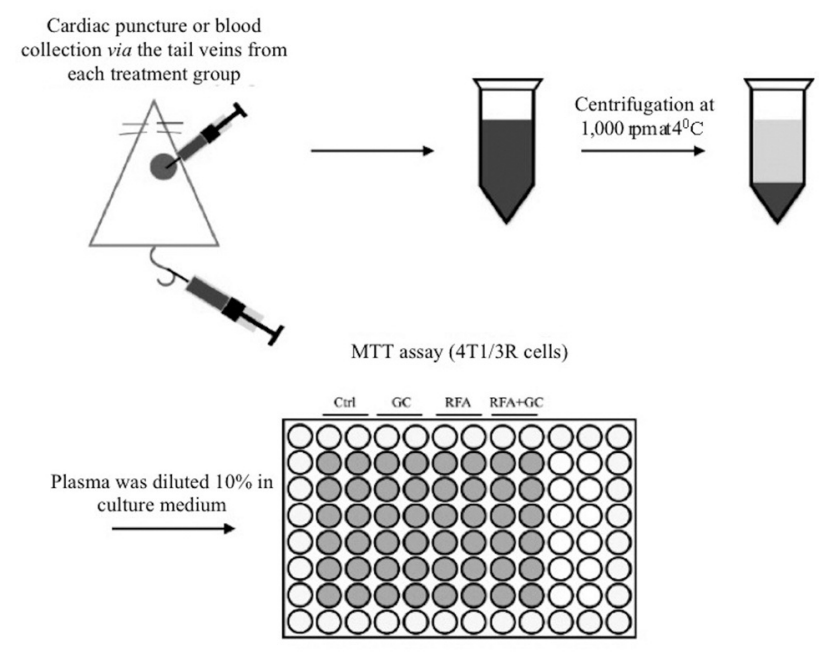

B

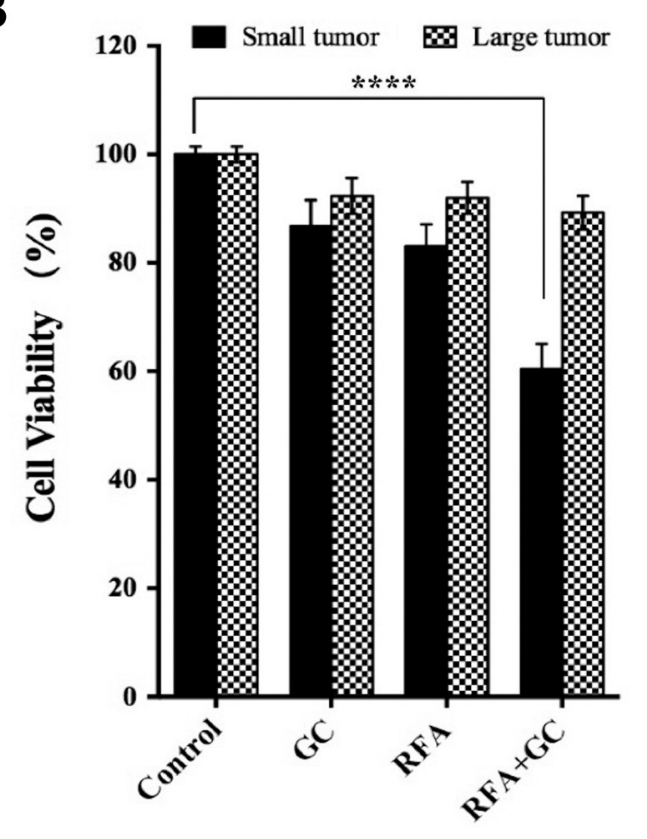

Figure 5. Cytotoxicity of blood plasma from GC and RFA treatments on $4 T 1$ cells. (A) Diagram of plasma extraction followed by MTT assay; (B) Comparison of cytotoxicity raised by plasma from small and large tumors treated with different methods. $* * * * p<0.0001$. GC, Glycated chitosan; RFA, radiofrequency ablation.

remains to be studied. Since the cytokines affected by RFAGC combined treatment are associated with tumor control, it is plausible that GC may modulate the antitumor immunity following hyperthermia. Furthermore, the plasma of tumorbearing mice on inhibiting the tumor cell viability (Figure 5) indicated the possible generation of specific antibody(ies) by the RFA-GC treatment, which could lead to antibody- 
Control GC RFA RFA+GC
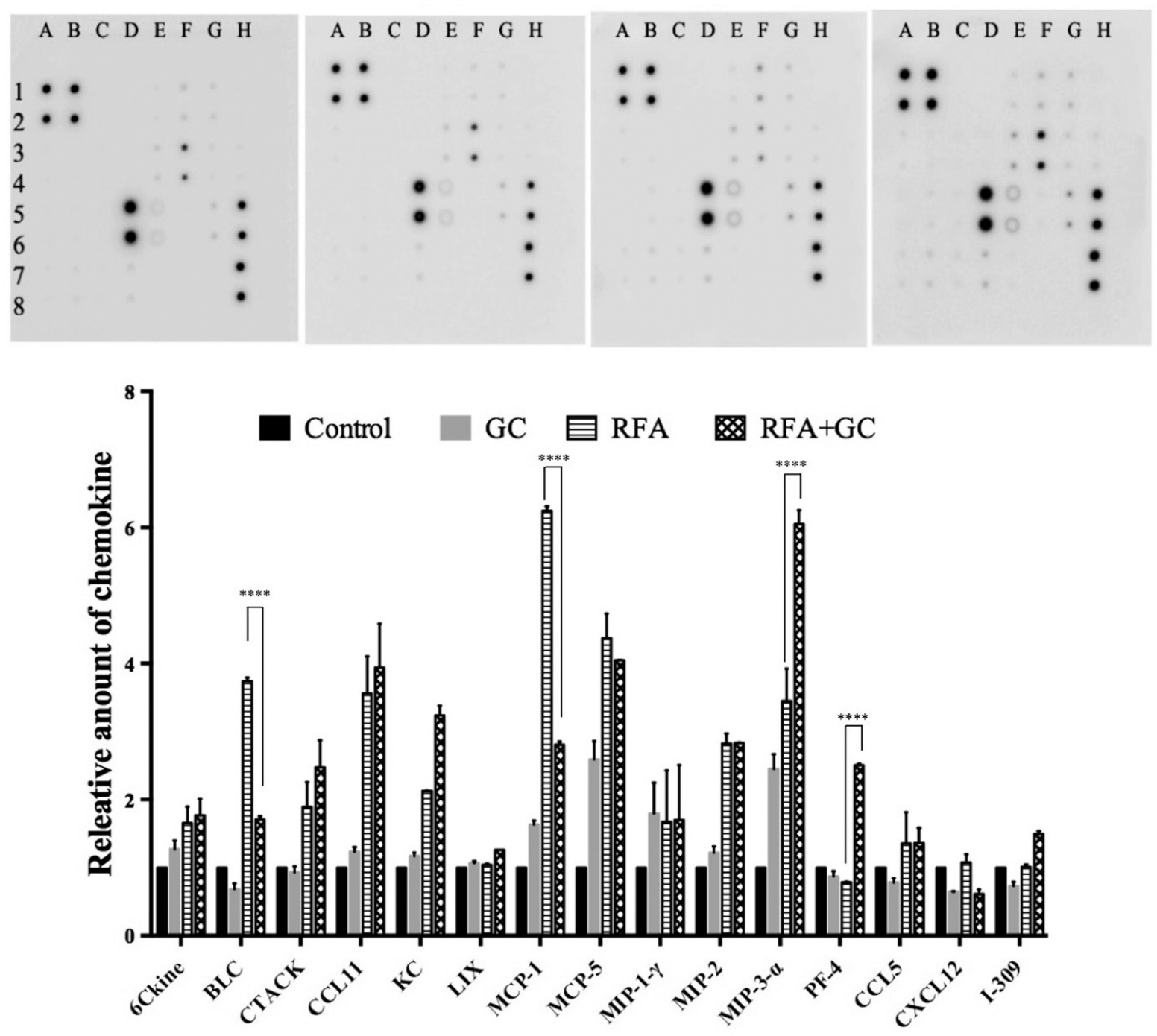

Figure 6. Chemokine array analysis from tumor-bearing mice treated with GC and RFA. (A) Blot of chemokine arrays (each cytokine was duplicated); (B) Quantification of dot signals on the array using ImageJ software with densitometric function. $* * * *<<0.0001$.

dependent complement-mediated cytotoxicity. It is possible the RFA-GC activated both cellular and humoral arms of the host immune responses. This will be an interesting topic in our future studies, such as ELISA-based detection of the difference in $\operatorname{IgM}$ and $\operatorname{IgG}$ antibody titers.

Additionally, although photothermal application can release a large amount of different antigens for the generation of antitumor immunity, the response of the host immune system is limited $(49,50)$. When combined with GC, CD80 expression on dendritic cells (DCs) could be significantly enhanced, so could be the antigen acquisition and antigen presentation by antigen presenting cells (APCs), thus activating cytotoxic Tlymphocytes (CTLs) (50). Therefore, immunologic intervention is required to invoke the immune system to achieve an effective and protective immune response against residual tumor cells. Whether GC combining RFA can also induce $\mathrm{CD}^{+}$T-cell activation, related mechanisms should be important to clarify in the future, i.e. the levels of innate and humoral immune responses for tumor suppression.

The limitation of this study is that the temperature recorded by RFA was based on the hooked electrode rather than the tumor mass per se. It is not surprising to see that small tumors 
responded better than large tumors to treatment because the temperature distribution of small tumors should be greater even than that of larger tumors after RFA treatment. Furthermore, our results also provide evidence that RFA-GC, like any modality, loses its potential when tumor burden becomes too big, simply because of the limitation of any treatment method in controlling an overwhelming tumor burden. Without effective control of the primary tumor, the immune system may not be strong enough to control metastasis. Although we used mild hyperthermia and short temperature exposure time, we also tried longer exposure time (60 seconds) under the same temperature range. However, the results were not improved using RFA alone (data not shown). The pain of animals, according to their body responses, seemed also enhanced at longer temperature exposure. Therefore, the current regime of RFA should be ideal for other tumor models using immune competent animals in the future.

\section{Conclusion}

The current data suggest that the immunoadjuvant GC can enhance the therapeutic efficacy of hyperthermia in small breast tumors. Use of a syngeneic, immune competent tumor model demonstrated that RFA-induced immune responses could be modulated by GC. The combination of RFA and GC not only eradicated tumor but also suppressed metastasis in vivo. As GC has been proven to be a non-toxic agent and RFA has been used in clinical settings, our results may provide a novel potential strategy to combine these methods for human tumor control in the future.

\section{Acknowledgements}

This study was supported, in part, by Minister of Science and Technology of Taiwan (MOST 105-2628-B-010-013-MY3) and Taipei City Hospital, RenAi Branch (No.16). Additionally, the grants of US National Institutes of Health (R21 EB0155091-01 and RS20132225-106) and the Oklahoma Center for Advancement of Science and Technology (HR16-085) also contributed to related research. The Authors also thank the Taiwan Mouse Clinic (MOST 104-2325-B-001-011) for technical support of the in vivo imaging experiment.

\section{References}

1 Hurwitz $M$ and Stauffer P: Hyperthermia, radiation and chemotherapy: The role of heat in multidisciplinary cancer care. Semin Oncol 41: 714-729, 2014.

2 Kampinga HH and Dikomey E: Hyperthermic radiosensitization: Mode of action and clinical relevance. Int J Radiat Biol 77: 399408, 2001.

3 Datta NR, Rogers S, Ordonez SG, Puric E and Bodis S: Hyperthermia and radiotherapy in the management of head and neck cancers: A systematic review and meta-analysis. Int $\mathbf{J}$ Hyperthermia 32: 31-40, 2016.
4 Rao W, Deng ZS and Liu J: A review of hyperthermia combined with radiotherapy/chemotherapy on malignant tumors. Crit Rev Biomed Eng 38: 101-116, 2010.

5 Datta NR, Rogers S, Klingbiel D, Gomez S, Puric E and Bodis S: Hyperthermia and radiotherapy with or without chemotherapy in locally advanced cervical cancer: A systematic review with conventional and network meta-analyses. Int J Hyperthermia 32(7): 809-821, 2016.

6 Snider JW, 3rd, Datta NR and Vujaskovic Z: Hyperthermia and radiotherapy in bladder cancer. Int J Hyperthermia 32: 398-406, 2016.

7 Brown DB: Concepts, considerations, and concerns on the cutting edge of radiofrequency ablation. J Vasc Interv Radiol 16: 597-613, 2005.

8 Nguyen T, Hattery E and Khatri VP: Radiofrequency ablation and breast cancer: A review. Gland Surg 3: 128-135, 2014.

9 McDermott S and Gervais DA: Radiofrequency ablation of liver tumors. Semin Intervent Radiol 30: 49-55, 2013.

10 Jeffrey SS, Birdwell RL, Ikeda DM, Daniel BL, Nowels KW, Dirbas FM and Griffey SM: Radiofrequency ablation of breast cancer: First report of an emerging technology. Arch Surg 134: 1064-1068, 1999.

11 Howenstein MJ and Sato KT: Complications of radiofrequency ablation of hepatic, pulmonary, and renal neoplasms. Semin Intervent Radiol 27: 285-295, 2010.

12 Zerbini A, Pilli M, Penna A, Pelosi G, Schianchi C, Molinari A, Schivazappa S, Zibera C, Fagnoni FF, Ferrari C and Missale G: Radiofrequency thermal ablation of hepatocellular carcinoma liver nodules can activate and enhance tumor-specific T-cell responses. Cancer Res 66: 1139-1146, 2006.

13 Napoletano C, Taurino F, Biffoni M, De Majo A, Coscarella G, Bellati F, Rahimi H, Pauselli S, Pellicciotta I, Burchell JM, Gaspari LA, Ercoli L, Rossi P and Rughetti A: RFA strongly modulates the immune system and antitumor immune responses in metastatic liver patients. Int J Oncol 32: 481-490, 2008.

14 Montomoli E, Piccirella S, Khadang B, Mennitto E, Camerini $\mathrm{R}$ and De Rosa A: Current adjuvants and new perspectives in vaccine formulation. Expert Rev Vaccines 10: 1053-1061, 2011.

15 Kafetzopoulos D, Martinou A and Bouriotis V: Bioconversion of chitin to chitosan: Purification and characterization of chitin deacetylase from Mucor rouxii. Proc of the Natl Acad Sci USA 90: 2564-2568, 1993.

16 Chen WR, Carubelli R, Liu H and Nordquist RE: Laser immunotherapy: A novel treatment modality for metastatic tumors. Mol Biotechnol 25: 37-44, 2003.

17 Song S, Zhou F, Nordquist RE, Carubelli R, Liu H and Chen WR: Glycated chitosan as a new non-toxic immunological stimulant. Immunopharmacol Immunotoxicol 31: 202-208, 2009.

18 Zhou F, Song S, Chen WR and Xing D: Immunostimulatory properties of glycated chitosan. J X-ray Sci Technol 19: 285-292, 2011.

19 Chen WR, Adams RL, Carubelli R and Nordquist RE: Laserphotosensitizer assisted immunotherapy: a novel modality for cancer treatment. Cancer Lett 115: 25-30, 1997.

20 Chen WR, Korbelik M, Bartels KE, Liu H, Sun J and Nordquist RE: Enhancement of laser cancer treatment by a chitosanderived immunoadjuvant. Photochem Photobiol 81: 190-195, 2005. 
21 Chen WR, Liu H, Ritchey JW, Bartels KE, Lucroy MD and Nordquist RE: Effect of different components of laser immunotherapy in treatment of metastatic tumors in rats. Cancer Res 62: 4295-4299, 2002.

22 Chen WR, Singhal AK, Liu H and Nordquist RE: Antitumor immunity induced by laser immunotherapy and its adoptive transfer. Cancer Res 61: 459-461, 2001.

$23 \mathrm{Xu} \mathrm{F}$, Liu H, Wu X, Jiang H, Nordquist RE and Chen WR: Measurement of $\mathrm{x}$-ray attenuation coefficients of aqueous solutions of indocyanine green and glycated chitosan. Med Physics 26: 1371-1374, 1999.

24 Chen YL, Wang CY, Yang FY, Wang BS, Chen JY, Lin LT, Leu JD, Chiu SJ, Chen FD, Lee YJ and Chen WR: Synergistic effects of glycated chitosan with high-intensity focused ultrasound on suppression of metastases in a syngeneic breast tumor model. Cell Death Dis 5: e1178, 2014.

25 Osorio FG, de la Rosa J and Freije JM: Luminescence-based in vivo monitoring of NF-kappaB activity through a gene delivery approach. Cell Commun Signal 11: 19, 2013.

26 Hayashi AH, Silver SF, van der Westhuizen NG, Donald JC, Parker C, Fraser S, Ross AC and Olivotto IA: Treatment of invasive breast carcinoma with ultrasound-guided radiofrequency ablation. Am J Surg 185: 429-435, 2003.

27 Burak WE, Jr., Agnese DM, Povoski SP, Yanssens TL, Bloom KJ, Wakely PE and Spigos DG: Radiofrequency ablation of invasive breast carcinoma followed by delayed surgical excision. Cancer 98: 1369-1376, 2003.

28 Medina-Franco H, Soto-Germes S, Ulloa-Gomez JL, RomeroTrejo C, Uribe N, Ramirez-Alvarado CA and Robles-Vidal C: Radiofrequency ablation of invasive breast carcinomas: A phase II trial. Ann Surg Oncol 15: 1689-1695, 2008.

29 Motoyoshi A, Noguchi M, Earashi M, Zen Y and Fujii H: Histopathological and immunohistochemical evaluations of breast cancer treated with radiofrequency ablation. J Surg Oncol 102: 385-391, 2010.

30 Yamamoto N, Fujimoto H, Nakamura R, Arai M, Yoshii A, Kaji $\mathrm{S}$ and Itami M: Pilot study of radiofrequency ablation therapy without surgical excision for T1 breast cancer: Evaluation with MRI and vacuum-assisted core needle biopsy and safety management. Breast Cancer 18: 3-9, 2011.

31 Mackey A, Feldman S, Vaz A, Durrant L, Seaton C and Klimberg VS: Radiofrequency ablation after breast lumpectomy added to extend intraoperative margins in the treatment of breast cancer (ABLATE): A single-institution experience. Ann Surg Oncol 19: 2618-2619, 2012

32 Majima H, Kashiwado K, Egawa S, Suzuki N and Urano M: Interaction between the kinetics of thermotolerance and effect of cis-diamminedichloroplatinum(II) or bleomycin given at 37 or 43 degrees C. Int J Hyperthermia 8: 431-442, 1992.

33 van der Zee J: Heating the patient: a promising approach? Ann Oncol 13: 1173-1184, 2002.

34 Zhang T, Pan Q, Xiao S, Li L and Xue M: Docetaxel combined with intraperitoneal hyperthermic perfusion chemotherapy and hyperthermia in the treatment of advanced ovarian cancer. Oncol Lett 11: 3287-3292, 2016.

$35 \mathrm{Hu} \mathrm{R}$, Ma S, Ke X, Jiang H, Wei D and Wang W: Effect of interleukin-2 treatment combined with magnetic fluid hyperthermia on Lewis lung cancer-bearing mice. Biomed Rep 4: 59-62, 2016.

36 Zhou L, Zhang M, Fu Q, Li J and Sun H: Targeted near infrared hyperthermia combined with immune stimulation for optimized therapeutic efficacy in thyroid cancer treatment. Oncotarget 7: 6878-6890, 2016.

37 Bergs JW, Franken NA, Haveman J, Geijsen ED, Crezee J and van Bree C: Hyperthermia, cisplatin and radiation trimodality treatment: A promising cancer treatment? A review from preclinical studies to clinical application. Int J Hyperthermia 23: 329-341, 2007

38 Skitzki JJ, Repasky EA and Evans SS: Hyperthermia as an immunotherapy strategy for cancer. Curr Opin Investig Drugs 10: 550-558, 2009

39 Toraya-Brown S and Fiering S: Local tumour hyperthermia as immunotherapy for metastatic cancer. Int J Hyperthermia 30: 531-539, 2014

40 Issels R, Kampmann E, Kanaar R and Lindner LH: Hallmarks of hyperthermia in driving the future of clinical hyperthermia as targeted therapy: Translation into clinical application. Int $\mathrm{J}$ Hyperthermia 32: 89-95, 2016.

41 Sottile ML, Losinno AD, Fanelli MA, Cuello-Carrion FD, Montt-Guevara MM, Vargas-Roig LM and Nadin SB: Hyperthermia effects on Hsp27 and Hsp72 associations with mismatch repair (MMR) proteins and cisplatin toxicity in MMRdeficient/proficient colon cancer cell lines. Int J Hyperthermia 31: 464-475, 2015

42 Calderwood SK and Ciocca DR: Heat shock proteins: stress proteins with Janus-like properties in cancer. Int J Hyperthermia 24: 31-39, 2008

43 R Rybinski M, Szymanska Z, Lasota S and Gambin A: Modelling the efficacy of hyperthermia treatment. J R Soc Interface 10: 20130527, 2013.

44 Tompkins WA, Rama Rao GV, Pantasatos P and Cain CA: Hyperthermia enhancement of antibody-complement cytotoxicity for human colon tumor cells. J Natl Cancer Inst 66: 453-459, 1981.

45 Chen L, Huang Z, Yao G, Lyu X, Li J, Hu X, Cai Y, Li W, Li X and $\mathrm{Ye} \mathrm{C}$ : The expression of CXCL13 and its relation to unfavorable clinical characteristics in young breast cancer. $\mathrm{J}$ Transl Med 13: 168, 2015.

46 Yoshimura T, Howard OM, Ito T, Kuwabara M, Matsukawa A, Chen K, Liu Y, Liu M, Oppenheim JJ and Wang JM: Monocyte chemoattractant protein-1/CCL2 produced by stromal cells promotes lung metastasis of $4 \mathrm{~T} 1$ murine breast cancer cells. PLoS One 8: e58791, 2013.

47 Choi Y and Kim CW: Antitumor effects of combined granulocyte macrophage colony stimulating factor and macrophage inflammatory protein-3 alpha plasmid DNA. Cancer Sci 101: 2341-2350, 2010.

48. Bikfalvi A: Platelet factor 4: An inhibitor of angiogenesis. Semin Thromb Hemost 30: 379-385, 2004.

49 Zhou F, Wu S, Song S, Chen WR, Resasco DE and Xing D: Antitumor immunologically modified carbon nanotubes for photothermal therapy. Biomaterials 33: 3235-3242, 2012.

50 Zhou F, Li X, Naylor MF, Hode T, Nordquist RE, Alleruzzo L, Raker J, Lam SS, Du N, Shi L, Wang X and Chen WR: InCVAX - A novel strategy for treatment of late-stage, metastatic cancers through photoimmunotherapy induced tumor-specific immunity. Cancer Lett 359: 169-177, 2015.

Received March 3, 2017

Revised April 17, 2017

Accepted April 18, 2017 